\title{
Neural changes after training with transcranial direct current stimulation to increase speech fluency in adults who stutter
}

\author{
Jennifer Chesters, ${ }^{1,2}$ Riikka Möttönen, ${ }^{1,3}$ and Kate E. Watkins ${ }^{1}$
}

\begin{abstract}
In a randomised controlled trial, we showed that a five-day intervention combining anodal transcranial direct current stimulation over the left inferior frontal cortex with temporary speech fluency enhancing techniques reduces stuttering. Speech fluency was unchanged by the fluency training alone, as predicted. Here, we report the neural changes associated with the intervention, measured using functional MRI during sentence reading before the training and one-week later.
\end{abstract}

We obtained imaging data in 25 adult men who stutter (median age $=32 \mathrm{y}$, inter-quartile range $=11$ ) at the pre-intervention baseline and again one-week post-intervention. A control group of 15 adult men who do not stutter (median age $=30 \mathrm{y}$, inter-quartile range $=10$ ) and did not complete the intervention were scanned on one occasion. In a whole-brain analysis of perceptibly fluent sentence reading, we compared the change in task-evoked neural activity in the sub-group of men who stutter who had received active stimulation during the intervention ( $\mathrm{N}=13$ ) with those who had sham stimulation $(\mathrm{N}=12)$. We hypothesised that the combination of anodal stimulation over the left inferior frontal cortex and fluency-enhancing training would result in lasting change to the brain networks supporting fluent speech production. An additional region-of-interest analysis explored effects on basal ganglia nuclei, which are thought to have a key role in the casual mechanism of stuttering, and which we hypothesised would be engaged by the behavioural approach used during training (choral and metronome-timed speaking).

One week after the intervention, the group who had received active transcranial stimulation showed increased activity in speech-related brain regions, relative to the group who had received sham stimulation. Cortically, these changes were evident in left inferior frontal cortex (pars opercularis and orbitalis), anterior insula, anterior superior temporal gyrus, 
anterior cingulate cortex, and supplementary motor area. Subcortically, activation increased in the caudate nuclei and putamen bilaterally, and in right globus pallidus and thalamus. Together these regions form cortico-striatal-thalamo-cortical loops involved in the planning and initiation and control of speech movements.

Our findings reveal that the mechanism of action of the tDCS intervention involved increasing activity across the network involved in the production of fluent speech, indicating that tDCS can be used to promote neural plasticity to strengthen networks supporting natural fluency. This study advances the potential of using non-invasive brain stimulation to improve therapy efficacy for those people who stutter who choose to work on increasing fluency.

\section{Author affiliations:}

1 Department of Experimental Psychology and Wellcome Centre for Integrative Neuroimaging, University of Oxford, OX2 6GG, UK

2 Bristol Speech and Language Therapy Research Unit, North Bristol NHS Trust, BS10 5NB, UK

3 Cognitive Science, Department of Digital Humanities, University of Helsinki, Finland.

Correspondence to: Dr. Jennifer Chesters

Bristol Speech and Language Therapy Research Unit, North Bristol NHS Trust, Pines and Steps, Southmead Hospital, Westbury-on-Trym, Bristol, BS10 5NB, UK

jennifer.chesters@nbt.nhs.uk

Running title: Neural change after tDCS for stuttering

Keywords: developmental stuttering; transcranial direct current stimulation; inferior frontal cortex; basal ganglia; randomized controlled trial

Abbreviations: $\mathrm{ACC}=$ anterior cingulate cortex; $\mathrm{fMRI}=$ functional magnetic resonance imaging; IFC = inferior frontal cortex; PWS = person who stutters; PWS-A = person who 
stutters in active tDCS group; PWS-S = person who stutters in sham tDCS group; SMA = supplementary motor area; STG = superior temporal gyrus; tDCS = transcranial direct current stimulation ; RCT = randomised controlled trial; SSI-4 = Stuttering Severity Instrument, version 4.

\section{Introduction}

The ability to speak fluently is a highly valued social skill, so the impact of living with a stutter can be considerable and far-reaching, affecting education and employment, social interaction, mental health, and overall quality of life. ${ }^{1-5}$ Speech and language therapy options to support adults who stutter are varied. A person who stutters may choose to engage in intervention to increase speech fluency, to address psychological or social aspects of living with a stutter, or some combination of these approaches. ${ }^{6}$ Outcomes of therapeutic intervention can be limited for those adults who stutter who choose to work on increasing fluency, however. ${ }^{7}$ There can be considerable costs to speech naturalness, especially in the short term, and continual practice is required to maintain effects. For these reasons, we were interested in whether transcranial direct current stimulation could offer a way to increase the effects of behavioural fluency therapy.

We completed a randomized controlled trial with a group of adult men who stutter, which showed that stuttering can be reduced by transcranial direct current stimulation (tDCS) paired with a fluency intervention. Anodal tDCS was applied over left inferior frontal cortex (IFC) at $1 \mathrm{~mA}$ for five consecutive daily sessions of 20 minutes duration. In each session, we applied the tDCS while fluency was temporarily enhanced using metronome and choral speech tasks. These techniques have the benefit that they result in a striking and immediate increase in fluency. However, the effects do not persist when habitual speaking is resumed. Therefore, we were interested in whether tDCS could interact with the neural mechanisms supporting fluent speech induced by these temporary fluency enhancers, resulting in a lasting fluency benefit. Consistent with this prediction, we found that disfluency was reduced one and six weeks after the intervention, relative to pre-intervention levels, for the group of people who stutter receiving active tDCS, but not for the group receiving sham stimulation. ${ }^{8}$ Both groups received the temporary fluency enhancement. The assignment of participants to receive 
anodal or sham stimulation was concealed from the participants and the researcher completing the intervention, assessment, and data analyses.

Since we were interested in the neural changes in speech production networks underlying changes in stuttering behaviour, the RCT participants also completed a speech task during functional magnetic resonance imaging (fMRI), before and one week after the intervention. The outcomes of this fMRI measure are reported here. Previous functional imaging studies have identified differences in activation in speech-related brain areas between people who stutter and people who do not stutter during both tasks and task-free resting-state scans. ${ }^{9-12}$ Several studies of brain activity in people who stutter make a distinction between measuring 'trait' and 'state' characteristics of stuttering. ${ }^{10,13,14}$ Trait characteristics are the neural features that distinguish people who stutter, even when speaking fluently, from people who do not stutter. In other words, they are the features of a speech network that is more vulnerable to disruptions in fluency. In contrast, 'state' characteristics are the patterns of brain activity present during actual moments of stuttering. Our tDCS intervention was designed to reinforce the brain network underpinning fluent speech, so the key neural changes supporting a lasting increase in fluency for people who stutter would involve 'trait' characteristics. Specifically, a shift in brain activity patterns towards the pattern seen in people who do not stutter would indicate that the speech network is functioning to support fluency and decrease the likelihood of stuttered moments occurring. Collecting fMRI data during fluent speech allowed us to test for a change in brain activation towards this 'fluent mode', without the confound of stuttering 'state' related activity.

We chose to stimulate the IFC in our tDCS intervention as this is a key region for speech motor control, which shows altered activation in developmental stuttering. Left IFC activity is reduced in people who stutter when speaking, compared with people who do not ${ }^{9,15-18}$ and the integrity of white matter tracts connected to the left IFC is disrupted. ${ }^{9,19,20}$ Diffusion tensor imaging and tractography has revealed that white matter tracts affected in stuttering sit within the arcuate fasciculus and branches of the superior longitudinal fasciculus, connecting parts of the left IFC (pars opercularis and ventral premotor cortex) with left ventral sensorimotor cortex, supramarginal and angular gyri, and middle and superior temporal gyrus. ${ }^{21} \mathrm{~A}$ consequence of this white matter disruption is a reduction in co-ordinated activity 
between regions connected by these tracts. During speaking, functional connectivity is reduced in people who stutter relative to those who do not, between left pars opercularis and inferior pre-central gyri bilaterally, ${ }^{22}$ right middle and superior temporal gyrus, supramarginal gyrus, ${ }^{23}$ and left inferior parietal lobule. ${ }^{24}$ Left-lateralised excitability of inferior pre-central gyrus (oro-facial motor cortex) is reduced in people who stutter relative to people who do not stutter during speech planning, as indexed by electromyographic recordings of potentials evoked by transcranial magnetic stimulation. ${ }^{24}$ This is consistent with less efficient communication between the left IFC and sensorimotor cortex. ${ }^{24,25}$ In addition, reduced auditory cortical activation during speech has been described as a 'neural signature' of developmental stuttering in functional imaging meta-analyses, ${ }^{13,14,26}$ and increased activation of the superior temporal cortex, particularly in the left hemisphere, is associated with successful fluency therapy. ${ }^{17,27}$ We hypothesised that applying tDCS over the left IFC cortex during fluent speaking would facilitate plasticity in the speech network, including the left IFC itself and these connected cortical regions, resulting in a lasting increase in fluency.

The speech motor control differences in developmental stuttering share some characteristics with the effects on movement initiation experienced by people with Parkinson's Disease, and other motor control conditions that implicate the basal ganglia circuits, dopamine, or both. ${ }^{28-}$ ${ }^{31}$ For example, difficulty initiating speech sounds, accompanied by increased tension in the vocal tract (described as "blocking") is one feature of stuttered speech that can be particularly uncomfortable. We were particularly interested, therefore, in the role of the basal ganglia in successful tDCS intervention. In fact, difference in basal ganglia function has been suggested as a core feature in developmental stuttering. ${ }^{28,32}$ People who stutter show alterations in taskrelated activity in the caudate nuclei and putamen, which form the dorsal striatum of the basal ganglia ${ }^{9,16,18,33}$ and severity of stuttering symptoms correlates with basal ganglia activity (specifically in the caudate nuclei bilaterally). ${ }^{33}$ Matching speech to an external signal, as used in our RCT, can dramatically reduce stuttering, ${ }^{34,35}$ and also modulates basal ganglia activation. 16,18 This effect parallels the beneficial effect of sensory guidance on limb movement in people with Parkinson's Disease, such as using visual stimuli on the floor ${ }^{30}$ or an auditory beat ${ }^{36}$ to support walking. A previous behavioural study in which metronome speech was practised daily over eight weeks, resulted in a pre-intervention underactivity in putamen and caudate nuclei being abolished. ${ }^{27}$ Here, we hypothesised that tDCS would consolidate the 
effects of a much shorter intervention with temporary fluency-enhancers, and similarly would modulate activity levels in the dorsal striatum.

In summary, we were interested in how anodal tDCS over the left IFC during a 5-day fluency intervention induces lasting effects on the functioning of cortical and subcortical brain areas supporting fluent speech. We assessed change in brain activity from pre-intervention baseline to one week after the intervention. At this time-point, the group who had received active tDCS were stuttering less during speech measured outside of the scanner, while the sham group showed no change in stuttering. We hypothesised that the reduction in stuttering following the tDCS intervention would be associated with increased activity in the left IFC, and modulation of connected cortical and subcortical regions engaged during speaking. Therefore, we predicted that the group who had received active tDCS would show a significantly greater change in brain activity in these brain regions one week postintervention, than the group who had received sham stimulation.

\section{Materials and methods}

\section{Participants}

Twenty-five adult men who stutter completed an MRI scan prior to, and one week following, a 5-day intervention using anodal tDCS over inferior frontal cortex, or sham stimulation, paired with temporary fluency-enhancing speech tasks. Thirty participants completed the speech outcome measures for the RCT (which were the primary outcomes). A schematic of the full study is shown in figure 1. However, five of these participants were unable to complete the MRI part of the study due to scanning contra-indications, discomfort in the scanner, or inability to attend for the scans. Exclusion criteria included any disorder of speech, language or communication other than developmental stuttering, sensory impairment, neurological or psychiatric illness, use of medications that act of the central nervous system, and any safety contra-indication for tDCS (e.g. personal or family history of seizures, taking medications or substances known to alter seizure threshold). Participants were classified as 
having at least a moderate stutter severity using the Stuttering Severity Instrument, version $4(\mathrm{SSI}-4)^{37}$ (table 1), and were randomly assigned to receive anodal tDCS of left inferior frontal cortex (Person who stutters - anodal stimulation: PWS-A group, $n=13$ ) or sham tDCS (Person who stutters - sham stimulation: PWS-S group, $n=12$ ). Fifteen adult men who do not stutter were also scanned on one occasion as a control group. Participants were recruited from the UK general population via online advertisement on social media, and the British Stammering Association website. All participants were right-handed. Groups were well matched on age and education (see table 1). The University of Oxford Central University Research Ethics Committee (MSD-IDREC-C2-2014-013) approved the study. Participants gave informed written consent to participate in the study, in accordance with the Declaration of Helsinki, and with the procedure approved by the committee. The trial was registered on ClinicalTrials.gov (NCT02288598).

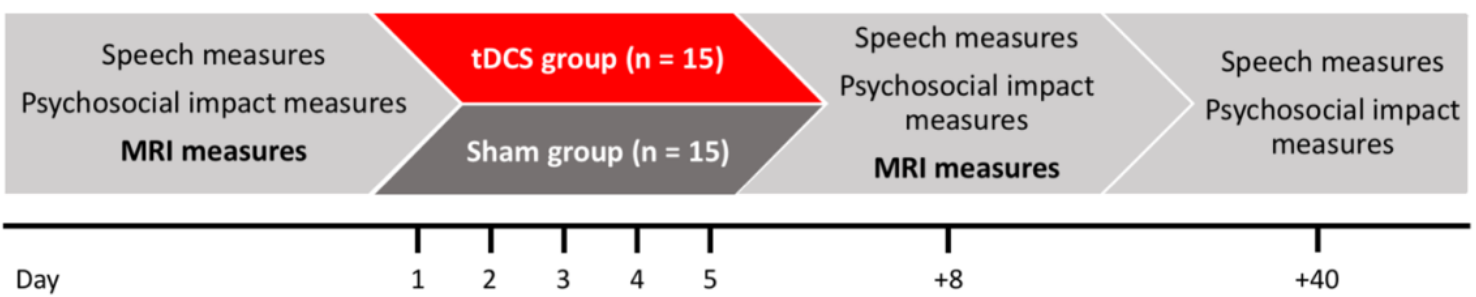

Baseline

Intervention

Follow up

Figure 1: schematic of full study design. 30 participants were enrolled into the randomised controlled trial. 25 of the 30 participants completed the MRI outcomes measures (five participants were unable to complete the MRI part of the study due to scanning contra-indications, discomfort in the scanner, or inability to attend for the scans). MRI scans were completed at baseline and one week (8 days on average) after the end of the intervention. Participants were randomly assigned to receive tDCS or sham stimulation during intervention.

Table I Demographic characteristics

\begin{tabular}{|c|c|c|c|c|}
\hline & \multicolumn{3}{|c|}{ PWS } & \multirow{2}{*}{$\begin{array}{c}\text { PWNS } \\
n=15\end{array}$} \\
\hline & PWS-A $(n=13)$ & PWS-S $(n=12)$ & $\begin{array}{c}\text { All PWS } \\
(n=25)\end{array}$ & \\
\hline Age in years & $35(13)$ & $30.5(\mathrm{II})$ & $32(11)$ & $30(10)$ \\
\hline $\begin{array}{l}\text { Years in } \\
\text { education }\end{array}$ & $17(3)$ & $16.5(3.5)$ & $17(4)$ & $17(1.5)$ \\
\hline SSI-4 score & $27(9)$ & $27.5(3)$ & $27(6)$ & $\mathbf{n} / \mathbf{a}$ \\
\hline
\end{tabular}

Median (inter-quartile range) scores are reported for all measures. Scores are reported for the whole group of people who stutter (All PWS) and people who do not stutter (PWNS), as well as for the subgroups of PWS who received active transcranial stimulation (PWS-A) and those who received sham stimulation (PWS-S). Stuttering Severity Instrument (SSI-4) scores range from 0-56, with higher scores indicating greater severity 


\section{Data acquisition}

Functional MRI data were obtained at the Oxford Centre for Clinical Magnetic Resonance Research (University of Oxford), using a 3T Siemens Trio scanner with a 32-channel head coil. Structural images were acquired at 1-mm isotropic resolution using a T1-weighted MPRAGE sequence $\left[\right.$ repetition time $(T R)=2040 \mathrm{~ms}$, echo time $(T E)=4.7 \mathrm{~ms}$, flip angle $=90^{\circ}, 192$ transverse slices, fat suppression].

Functional imaging data were acquired using sparse sampling, allowing participants to speak in the absence of scanner noise and to clearly hear the auditory task stimuli. Whole-head echo-planar images (TE = $30 \mathrm{~ms}$ ) were acquired every $9 \mathrm{~s}$ with a silent delay of $7 \mathrm{~s}$ (i.e., sparse sampling) and comprised 2-s acquisition of $383.5 \mathrm{~mm}$ interleaved axial slices (in plane resolution $3 \times 3 \mathrm{~mm}$ ). A $192 \mathrm{~mm}$ field of view was used, positioned to cover the cerebellum and brainstem. GRAPPA parallel imaging was used with an acceleration factor of 2. An anterior-posterior phase encode direction was used. A crosshair appeared in the middle of the screen during the 2-s acquisition period. During the 7-s silent delay between measurements, participants read aloud the text stimulus presented on the screen (or remained silent during rest trials). 96 volumes were collected, including 24 volumes for each speaking condition and for the rest condition; the task lasted 14.5 minutes in total. Speaking task volumes were collected in blocks of three, interspersed with a single volume of rest. The order of speaking conditions was fixed and pseudorandom. The field map was acquired with echo time difference of 2.46 seconds.

\section{Speech task}

The fMRI task involved reading sentences selected from the Harvard IEEE stimulus set ${ }^{38}$ aloud in a solo reading condition and in two conditions using fluency-enhancing techniques: metronome speech, and choral speech (figure 2). Stimuli were presented using NBS Presentation software version 18, back-projected and viewed via an angled-mirror mounted on the head coil. The task included 96 trials in total: 24 per speaking condition and 24 rest trials. During the solo reading condition, participants read sentences at a natural speech rate. 
In the Choral speech condition, participants heard a recording of a British male speaker reading the sentence, with an average speaking rate of 200 syllables per minute. Participants were instructed to read the sentence in time with the recorded voice. In the metronome speech condition, the sentence text was presented simultaneously with a $440 \mathrm{~Hz}$ pure tone at a 200 beats per minute rate, and participants were instructed to read the sentence in time with the tone. Participants were instructed to read the sentences aloud and to stop speaking when the crosshair appeared. This ensured that there would be no speech related movement of the head during data collection. Participants' speech was recorded using a fibre-optic microphone and two-track audio recorder. A baseline (rest) condition was also included for comparison, in which participants saw a string of unfamiliar non-native orthographic characters (Farsi) and were silent. Prior to the scan, participants practised the task until they were comfortable, and were able to perform the fluency enhancing speech conditions.

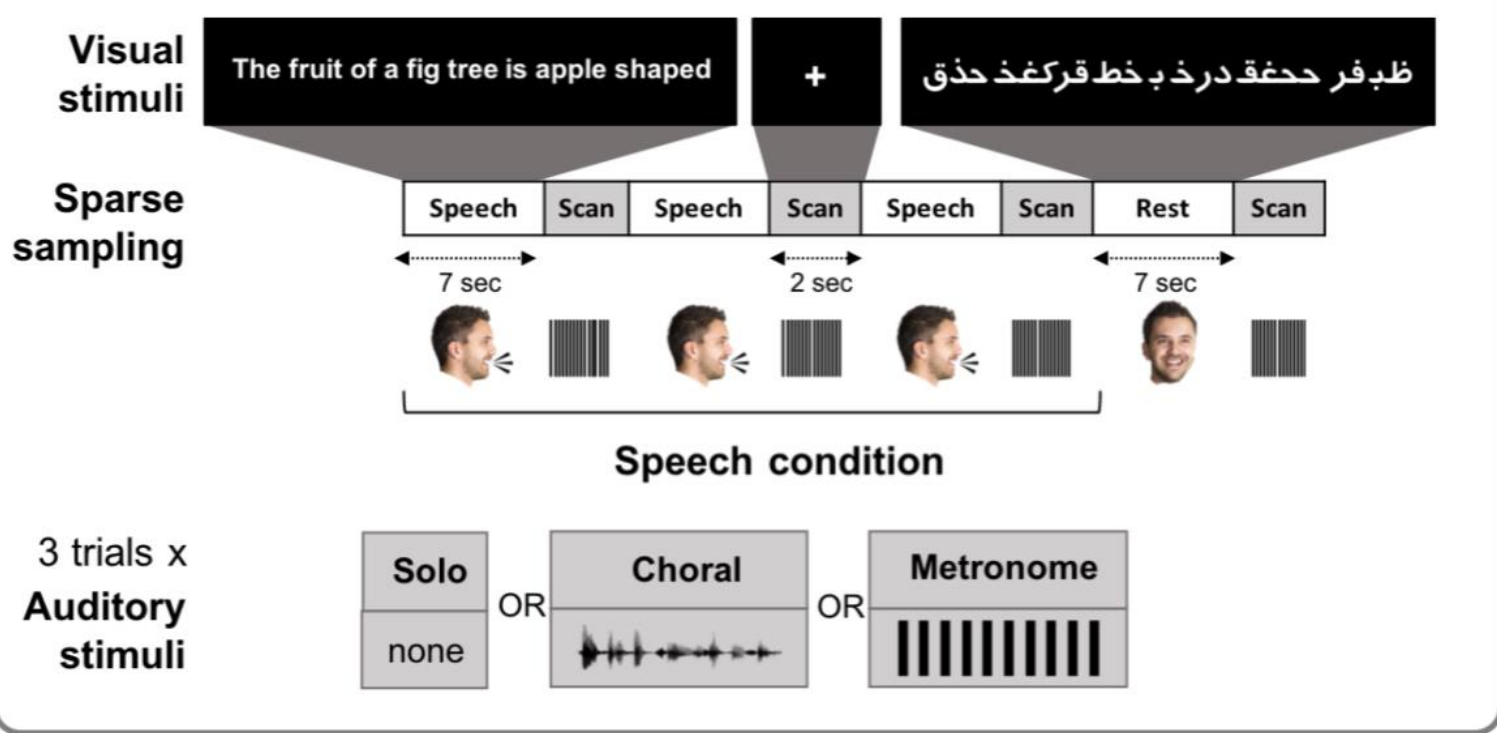

Figure 2: fMRI task design Participants read sentences in three speaking conditions: reading alone (solo), reading in time with a recorded voice (choral) and reading in time with a metronome at $200 \mathrm{bpm}$ (metronome). Rest conditions involved viewing non-native text while remaining silent. A sparse sampling design was used, so that participants spoke during a silent period between scans.

\section{Speech and $\mathrm{fMRI}$ analysis}

\section{Processing}

FMRI data processing was carried out using the general linear model at the individual level using FEAT (FMRI Expert Analysis Tool) Version 6.00, part of FSL (FMRIB's Software Library, 
www.fmrib.ox.ac.uk/fsl). Registration to high resolution structural and standard space images was carried out using FLIRT ${ }^{39,40}$ and non-brain removal using BET. ${ }^{41}$ We unwarped each data set using a fieldmap and PRELUDE and FUGUE software running in FSL and spatially smoothed with a 6-mm full-width-at-half-maximum smoothing kernel. We used grand-mean intensity normalisation of the entire $4 \mathrm{D}$ dataset by a single multiplicative factor and high pass temporal filtering (Gaussian-weighted least-squares straight line fitting, with sigma=45.0s).

We corrected for motion using standard motion correction in MCFLIRT, using the middle scan as reference. ${ }^{39}$ Manual checking was also used to identify trials with excessive motion (defined as $>3 \mathrm{~mm}$ ), however, no trials met this criterion. We included two regressors to account for physiological effects during the scan. These regressors were the mean timecourses extracted from pre-processed data from 3-mm radius spheres: one in cerebrospinal fluid of the anterior lateral ventricle (standard space coordinates $2,10,8$ ) and the other within white matter in the dorsal posterior frontal lobe $(-26,-22,28)$. These co-ordinates were taken from a previous study using this approach ${ }^{42}$. Participants' speech was recorded in the scanner and analysed for moments of stuttering. The group of men who stutter produced stuttering on some sentences during both the pre- and post-intervention scans. Sentences that contained any stuttering were regressed out, so that the analysis focussed only on fluent speech. At pre-intervention, 24 of the 25 participants stuttered during at least one sentence, with an average of 14 of the 72 sentences containing some stuttering. A post-intervention, 23 of the 25 participants stuttered during at least one sentence, with an average of 12 of the 72 sentences containing some stuttering. The control group of participants who do not stutter were fluent during all speaking conditions.

\section{Statistical analysis}

The sample size for the RCT was based on a power calculation from previous intervention trials of tDCS in aphasia, as no studies in developmental stuttering were published at that time. A sample size of 15 participants in each group, gave $80 \%$ power (with $p<0.05$, onetailed) to detect a large effect size (Cohen's $d>0.9$ ) for the main effect of stimulation.

Using the general linear model in FEAT, we modelled three contrasts of interest for each participant: the average for each speaking condition (Solo, Choral, Metronome) relative to 
the rest condition, and also contrasted these averages for each speaking condition against each other. We were centrally interested, however, in the differences between the groups and over time, across speaking conditions, so we included an F-test across Solo, Choral and Metronome conditions.

The fsl-randomise tool ${ }^{43}$ for non-parametric permutation inference was used to analyse individual COPE and F-statistic images at the group level. We used 5000 permutations to build a null distribution, variance smoothing of $2.55 \mathrm{~mm}$ (due to relatively small sample sizes), and the results were reported using threshold-free cluster enhancement. We used two-sample unpaired t-tests to investigate the differences in activation between the control group of people who do not stutter (PWNS) and the group of people who stutter (PWS) at preintervention baseline, before randomisation PWS-A and PWS-S sub-groups. We also ran onesample t-tests for the PWS and control groups separately to show the group average data across speaking conditions. The PWS and control groups did not show different patterns of activation between the three speaking conditions, so we focussed on our main question of the general effects on speaking using the first-level F-statistic images. To test whether the change in neural activation in the PWS-A group following intervention differed to that for PWS-S group, we first used fslmaths to subtract the pre-intervention from the postintervention F-stat map for each participant. We then compared the F-stat difference images between groups using a two-sample unpaired t-test. Additionally, we assessed the average difference between pre- and post-intervention for each group separately using one-sample ttests.

Finally, we were interested in activations in basal ganglia nuclei, that may have been modulated by the intervention. Activation in these small structures can be more difficult to pick up with a whole brain approach, so we extracted structurally defined regions of interest for the caudate nuclei and putamen bilaterally, using FIRST in FSL ${ }^{44}$ to segment these structures using the high-resolution T1-weighted image. Percentage BOLD signal change from rest was extracted for each speech condition using featquery in FSL, for each ROI. Group level analyses were performed in SPSS version 26 (IBM). To test for changes in BOLD activity related to the tDCS intervention, we calculated the difference in percent signal change (preintervention subtracted from post-intervention \% BOLD change from rest) for each person 
who stutters, within each ROI. We completed mixed model ANOVAs for the caudate nuclei and putamen, with a within-subjects factor of speaking condition (solo, choral, metronome), and hemisphere (left, right) and a between-subjects factor of group (PWS-A, PWS-S).

\section{Data availability}

Uncorrected maps of group differences in NIFTI format, and behavioural data regarding sentences produced fluently vs disfluently during the fMRI reading task, are available in NeuroVault: https://neurovault.org/collections/11951/. Audio recordings have not been made available, in line with ethics approval, as they are considered identifying information.

\section{Results}

\section{Activation at pre-intervention baseline}

Across all speaking conditions compared with the rest condition (viewing false font), the expected network of areas involved in reading sentences aloud and receiving sensory feedback of this speech was activated (supplementary fig. S1). Pre-intervention, both our group of men who stutter and our control group of men who do not stutter showed widespread brain activation. This included lateral sensorimotor cortex bilaterally extending to anterior insula and frontal opercular cortex more prominently on the left, and medially to the supplementary motor area (SMA) and cingulate motor areas. There was extensive activation of the superior temporal gyrus (STG) along its full extent and in both hemispheres. Subcortically, there was activation of the basal ganglia, thalamus, midbrain, and cerebellum, all bilaterally. In the group of men who stutter, activity in the medial occipital cortex was evident. The statistical maps for activity at pre-intervention baseline in both groups are available on Neurovault (see data availability section of methods).

Comparison of activation between the groups at pre-intervention baseline showed several regions of lower activity in the people who stutter. These included the right subcentral gyrus, a large portion of the medial parietal cortex extending to the paracentral lobule, the dorsal occipital cortex and portions of the anterior cerebellum. The group of people who stutter did 
not show higher activity than the control group in any region (figure 3, supplementary table S1). As we excluded any sentences containing stuttering, these neural differences were not driven by an overt behavioural difference between the groups.

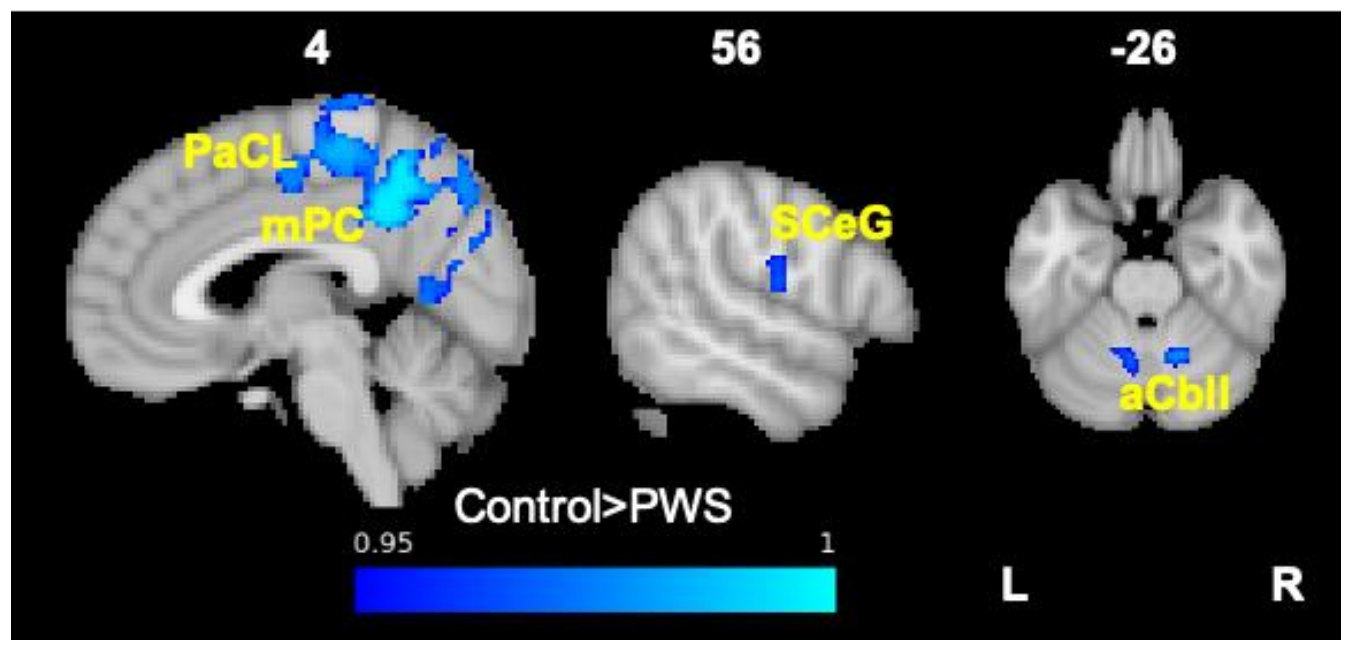

Figure 3: Lower activity in PWS group at pre-intervention baseline, compared to PWNS control group. The blue map shows the 1-p values for regions showing lower activity in the group of people who stutter (PWS) than the control group of people who do not stutter (PWNS), thresholded at $0.95(p=0.05)$. No regions showed higher activity in the PWS group. The map is overlaid on the MNI-152 average brain. Numbers next to each image indicate the coordinate in $\mathrm{mm}$ of that slice in $\mathrm{x}$ (for sagittal) and $\mathrm{z}$ (for axial). aCbll - anterior cerebellum, $\mathrm{PaCL}-$ paracentral lobule, mPC -medial parietal cortex, SCeG - subcentral gyrus.

\section{Change in activation related to the tDCS intervention}

The group of people who stutter who received anodal tDCS during the intervention (PWS-A) showed a significant increase in their speech fluency one week post-intervention, measured during reading and conversation outside of the scanner. ${ }^{8}$ Therefore, we were primarily interested in where in the brain the PWS-A group showed greater changes in activity between pre- and post-intervention scans relative to the group who received sham stimulation (PWSS), in which fluency was unchanged. We found that the PWS-A group showed significantly greater increases in neural activity following the intervention than the PWS-S group in a number of regions. These included the left inferior frontal cortex (pars orbitalis), left anterior cingulate cortex (cingulate motor area), left frontal opercular cortex extending medially to the anterior insula and laterally to the anterior STG, the right SMA, the right putamen and the globus pallidus bilaterally (figure 4; table 2). The PWS-S group did not show any greater changes in activation than the PWS-A group in any region. 
Table 2 Greater increase in activity following intervention in the PWS-A compared with the PWS$S$ group

\begin{tabular}{|c|c|c|c|c|c|}
\hline \multirow[t]{2}{*}{ Brain region } & \multirow{2}{*}{$\begin{array}{l}\text { Cluster } \\
\text { size } \\
\text { (voxels) }\end{array}$} & \multirow{2}{*}{$\begin{array}{l}\text { Peak voxel } \\
\text { I-p value }\end{array}$} & \multicolumn{3}{|c|}{ Co-ordinates of peak voxel } \\
\hline & & & $\mathbf{X}$ & $Y$ & $\mathbf{Z}$ \\
\hline $\begin{array}{l}\text { Left inferior frontal cortex, pars } \\
\text { orbitalis }\end{array}$ & 102 & 0.966 & -44 & 26 & -16 \\
\hline Left anterior cingulate cortex & 93 & 0.964 & -6 & 10 & 38 \\
\hline Left frontal opercular cortex & 651 & & & & \\
\hline anterior STG & & 0.989 & -48 & 6 & -8 \\
\hline anterior insula & & 0.988 & -38 & 6 & 0 \\
\hline Right lentiform nucleus & 519 & & & & \\
\hline putamen & & 0.988 & 22 & 2 & 8 \\
\hline \multirow[t]{2}{*}{ globus pallidus, pars externa } & & 0.980 & 22 & 2 & -2 \\
\hline & & 0.990 & 24 & -10 & 2 \\
\hline Right SMA & 165 & 0.966 & 2 & -6 & 66 \\
\hline Right anterior thalamus & 82 & 0.968 & 8 & -6 & -8 \\
\hline $\begin{array}{l}\text { Right posterior superior frontal sulcus } \\
\text { (junction with precentral sulcus) }\end{array}$ & 53 & 0.978 & 24 & -10 & 46 \\
\hline Left globus pallidus, pars interna & 39 & 0.964 & -22 & -14 & -6 \\
\hline Right dorsal precentral gyrus & 44 & 0.965 & 20 & -18 & 68 \\
\hline Right thalamus/parahippocampal gyrus & 38 & 0.964 & 16 & -28 & -8 \\
\hline
\end{tabular}

Selected subpeaks (italics) reported for larger clusters; only clusters with $>30$ voxels extent are reported for brevity. Full maps are available online.

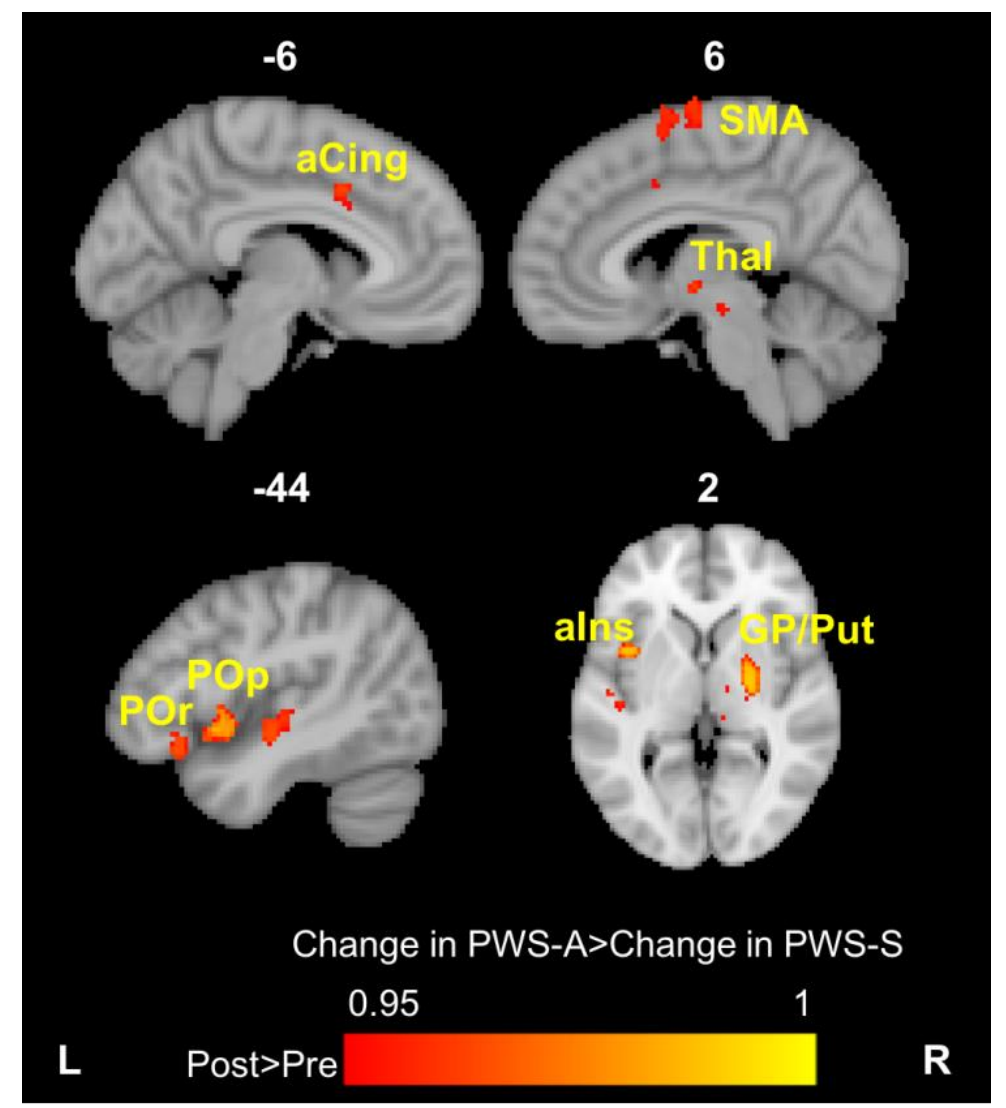

Figure 4: Change in activity following intervention, in the PWS-A compared with the PWS-S group: whole brain analysis. The red/yellow map shows the 1-p value map for regions showing greater activity change in the PWS-A compared with the PWS-S group, thresholded at $0.95(p=0.05)$. No regions showed greater change in the PWS-S than the PWS-A group. The map is overlaid on the MNI-152 average brain. Numbers above each image indicate the coordinate in $\mathrm{mm}$ of that slice in $x$ (for sagittal) and $z$ (for axial). aCing - anterior cingulate, alns - anterior insula, GP - globus pallidus, POp - pars opercularis, POr - pars orbitalis, Put putamen, SMA - supplementary motor area, Thal - thalamus.

Changes within the dorsal striatum in the PWS-A group were explored further using subjectspecific structurally defined ROIs. Consistent with the whole brain analysis, we found that the 
tDCS intervention significantly increased activity in the putamen, (main effect of stimulation: $F_{1,23}=9.09, p=.006$; no significant effect of hemisphere, figure 5). We also found increased activity in the caudate nucleus bilaterally (main effect of stimulation: $F_{1,23}=4.77, p=.039$, no significant effect of hemisphere, figure 5).

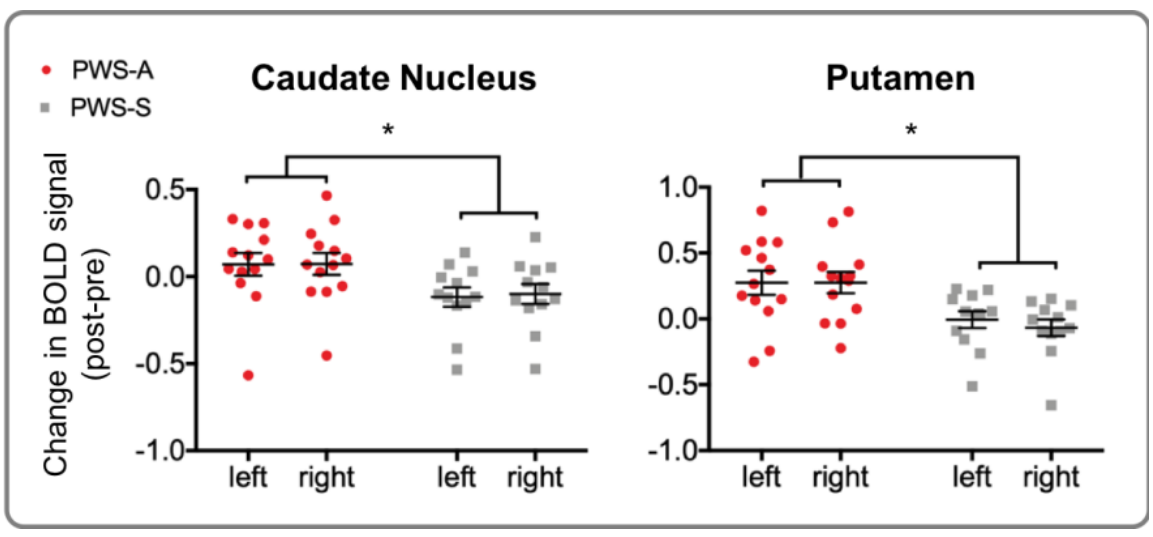

Figure 5: Change in activity following intervention, in the PWS-A compared to the PWS-S group: ROI analysis of caudate nucleus and putamen.

Individual data are plotted for the PWS-A group (red) and the PWS-S group (grey), showing change in BOLD signal in structurally defined ROls. relative to pre-intervention baseline. Group means indicated by black horizontal lines; error bars indicate standard error of the mean. Significant group differences are marked with a horizontal bar and asterisk.

\section{Discussion}

We previously completed an RCT of anodal tDCS over left IFC applied for 20 mins a day over 5 days, paired with a temporary fluency-enhancing training (using choral speech and metronome-timed speech). In a group of 30 men with moderate to severe stuttering, speech dysfluencies measured one week and six weeks after the intervention were reduced more in the 15 men randomised to receive tDCS than in the sham group ${ }^{8}$. Here, we explored the neural changes accompanying these changes in speech fluency, and found that one week after the intervention, the group who had received tDCS showed greater activity increases in speech-related cortical regions and subcortically in the striatum during speaking than the group who had received sham stimulation.

\section{Left IFC activity increased following the tDCS intervention}

We hypothesised that as the left IFC has a key role in producing fluent speech, electrical stimulation applied to left IFC would support the contribution of this region to the network of areas involved in fluent speech production. The anode was centred over pars opercularis, guided by the $10-10$ system for EEG electrode placement, ${ }^{38}$ and extended posteriorly to 
partially cover ventral portions of premotor and primary motor cortex. One week following the intervention, increased task-evoked activity was seen in the group who had received tDCS in cortical areas underneath the anode, including pars opercularis and the anterior insula.

These results are consistent with previous reports of increased left IFC activity during fluent speaking as a marker of a positive outcome of fluency therapy. ${ }^{17,45}$ Previous imaging work indicates reduced activity during speech production in left inferior frontal regions in PWS (e.g. left anterior insula and Rolandic operculum; ; left IFG; ${ }^{15}$ left ventral premotor cortex ${ }^{10,22}$ ). Neumann and colleagues ${ }^{17}$ reported that a three-week intensive fluency-shaping treatment program (Kassel Stuttering Therapy) resulted in reduced stuttering and increased activation during reading aloud in the left insula and Rolandic operculum, measured 12 weeks after the course, in the therapy maintenance phase. The portion of the IFC showing increased activity in our study overlapped with the region showing increased activity post-intervention in the Neumann study, which was centred on the left insula. In another study, PET scans taken before and immediately following a similar intensive fluency programme ${ }^{45}$ showed left lateralised activation in IFC and anterior insula, which was not present pre-therapy. This supports our hypothesis that tDCS can be used to improve therapy effectiveness, as we found changes in fluency and brain function following five sessions of tDCS-mediated behavioural intervention (totalling 1 hour 40 minutes) comparable to those reported 12 weeks following an intensive intervention (totalling approximately 90 hours).

\section{Activity across the cortical speech network increased following the tDCS intervention}

Brain areas connected to left IFC, across the network related to speech production, also showed increased task-evoked activity following our tDCS intervention. Specifically, we found increased activity in left auditory cortex, anterior cingulate cortex and pars orbitalis of the IFC, and in SMA, in the PWS-A group. Although tDCS is relatively non-focal (particularly given the large electrode size used here) these regions are unlikely to have received direct stimulation. Rather the effects on their activity would come about via connectivity with stimulated left IFC regions. 
Reduced auditory cortical activation in the left STG during speech production has been described as a 'neural signature' of developmental stuttering. ${ }^{13,14,26}$ Following intervention, the PWS-A group showed increased activity in this same part of the left STG, which also overlaps with activation change shown previously following a 3-week intensive course of fluency shaping therapy ${ }^{17}$.

Differences in SMA activity patterns have been highlighted as a marker for developmental stuttering, however the consistent pattern is one of relative overactivity. ${ }^{9,16,46}$ Theories that the causal mechanism in stuttering involves increased inhibition of speech movements ${ }^{47}$ or reduced ability to generate internal timing cues for speaking ${ }^{28}$ implicate the SMA/pre-SMA complex, and the SMA and anterior cingulate cortex (ACC), respectively. The SMA and ACC play a role in maintaining speech flow, including initiating ${ }^{48,49}$, and resolving conflict between motor plans to avoid speech errors. ${ }^{50}$ The ACC in particular is involved in volitional control to override overlearned responses. ${ }^{51}$ This is consistent with our finding of increased activation in both regions associated with a reduction of stuttering: the increased activation in the anterior cingulate cortex indicating a greater degree of cognitive control, and both regions supporting more effective monitoring and maintenance of fluency. The frontal aslant tract connects the inferior frontal cortex to the SMA; in the left hemisphere, this tract supports speech initiation and fluency, ${ }^{52}$, and reduced diffusivity within it has been associated with developmental stuttering. ${ }^{53}$

\section{Basal ganglia nuclei activity increased following the tDCS intervention}

We were specifically interested in exploring how the combination of tDCS with the behavioural intervention, which combined choral and metronome-timed speech, would affect activity in the dorsal striatum (caudate nucleus and putamen). An influential theoretical account of stuttering centres the function of the basal ganglia nuclei: ${ }^{28}$ a reduced ability to generate internal timing cues disrupts the network supporting internally cued movements, including outputs from the basal ganglia via the thalamus to SMA. Although not evident in the meta-analyses of functional images studies with people who stutter (possibly due to the small 
size of these structures), altered activation of caudate nuclei and putamen have been reliably reported. ${ }^{9,16,18,33,46} \mathrm{~A}$ recent study also revealed a higher iron content in the putamen of people who stutter, which is consistent with elevated dopamine levels. ${ }^{54}$ External cueing, e.g. by a metronome and during choral speech, increases basal ganglia activation in people who stutter. ${ }^{16,18}$ Structural equation modelling of fMRI data has revealed disrupted connectivity between inferior frontal gyrus bilaterally and the right putamen associated with speech planning in people who stutter. ${ }^{55}$

We investigated changes in basal ganglia activation in a region-of-interest analysis, in addition to our whole brain analysis, and found significant effects related to successful tDCS intervention with both approaches. In our whole brain analysis, we found increased activation in right globus pallidus pars externa, putamen, and thalamus. A topographic MRI imaging study using spectral analysis ${ }^{56}$ identified the more lateral and inferior parts of pallidum and putamen, where we see increased activation, contain representations of the mouth. Using participant-specific structurally-defined ROIs of the caudate nuclei and putamen, which were of particular interest $a$ priori, we found significant increases in mean activity in both structures bilaterally.

Investigations of the role of basal ganglia nuclei function, and of dopamine metabolism, in developmental stuttering reveal that this relationship is complex..$^{28,57}$ Dysfluency has been related both to increases and decreases of dopamine metabolism, which may reflect that dopamine availability should have a certain balance to support fluency, and that developmental stuttering could result from either excessively high or low levels. Increased fluency following fluency therapy has previously been associated with increased activation in left putamen ${ }^{58}$ and with the loss of a positive correlation between stutter severity and caudate nucleus activation. ${ }^{33}$ Our results are consistent with a previous therapeutic intervention that reduced stuttering in adults who stutter using metronome-timed speech daily over eight weeks; activity in globus pallidus, putamen and caudate nucleus of people who stutter increased to the same level as seen in people who do not stutter. ${ }^{27}$ Here, we found that tDCS can be combined with metronome-timed speech (as well as choral speech) to promote the same changes in a much shorter intervention period ( 1 hour 40 mins vs 10 hours minimum). Our finding of increased activation in the premotor cortex, putamen, 
thalamus, and SMA suggests that the combined intervention promotes co-ordination of functional connectivity within this sensorimotor circuit for self-initiated movement, which is hypothesised to be affected in developmental stuttering. ${ }^{28}$

\section{Lower neural activity at pre-intervention baseline in people who stutter}

At pre-intervention baseline, we found only regions of relative under-activity during fluent speech production in the men who stutter, compared with the control group of men who do not. This contrasts with meta-analyses reporting over-activity, particularly in right inferior frontal cortex and cerebellum, described as key 'signatures' of stuttering. ${ }^{13,26}$ One important methodological difference is that here we considered only speech produced fluently in both groups, so that we considered only brain activation differences associated with the 'trait' of being a person who stutters, rather than the 'state' (actual moments of dysfluency). Previous meta-analyses, however, combined findings from a range of studies using different speech tasks and both fluent and stuttered speech in people who stutter contrasted with people who do not. Although the later of the two meta-analyses ${ }^{13}$ reports a stuttering trait analysis, they did not exclude stuttered speech. When only the fluent speech of people who stutter is included in analysis, however, a pattern of exclusive under-activation relative to people who don't stutter has previously been reported, ${ }^{10}$ including in medial cerebellum and posterior cingulate cortex, which are replicated here. Our finding of lower activation in posterior cingulate cortex is consistent with an early PET study of stuttering, ${ }^{15}$ which also notably recruited people who stutter with relatively high SSI-4 scores.

Relatedly, we found only increases in activation following the tDCS intervention, which could have several potential explanations. Firstly, we were using anodal stimulation to upregulate activity in left IFC and connected brain regions. It is perhaps unsurprising that increased fluency was associated with up-regulation, rather than down-regulation, of a relatively underactive speech network. Secondly, previous research has shown differences between short and longer-term brain changes related to successful therapeutic outcomes for stuttering ${ }^{59}$ and other conditions (e.g. aphasia following stroke). ${ }^{60}$ For example, Neumann and colleagues found that fluency therapy alone resulted in an extensive pattern of increased 
activation in speech-related cortical regions (particularly in left hemisphere) during speaking, measured by fMRI immediately after therapy. At two year follow-up, the magnitude of these changes was reduced. ${ }^{59} \mathrm{~A}$ longer- term follow-up of the participants in this study would be needed to explore whether a similar pattern were revealed.

The men who stutter in our RCT had at least moderate stutter characteristics, scoring 25 or greater on $\mathrm{SSI}-4,{ }^{37}$ which meant our group had a higher average severity and a smaller variance than the cohorts of people who stutter studied in most previous imaging studies. For example, across the two recent meta-analysis, ${ }^{13,14}$ most studies included participants with stuttering classified as mild-moderate on average, and did not limit inclusion range, resulting in typical ranges from very mild to severe within a study. This may account for differences in results between previous studies, due to heterogeneity of stuttering characteristics. In addition, participants with 'milder' overt stuttering are likely over-represented, due to a positively skewed distribution of severity in the population of people who stutter. We chose a more stringent inclusion criteria in the RCT to increase group homogeneity and the potential for a measurable reduction in stuttering, to maximise the sensitivity of the study to the effects of tDCS. Therefore, our RCT cohort of people with higher SSI-4 scores likely represented a distinct sub-group both in terms of stuttering behaviours and underlying brain function.

It might be predicted that a group with more overt stuttering characteristics would display more robust differences in the regions consistently related to stuttering. However, it is important to consider the complexity of possible presentations of stuttering. For example, any individual who stutters is likely to experience a combination of overt and covert behaviours. Overtly 'mild' stuttering can involve compensatory strategies, such as word avoidance, use of synonyms, changing the speed or rhythm of speech, or re-planning sentence structures. In a group of people with stuttering classified as 'mild' by SSI-4, therefore, there is likely a combination of people experiencing fewer or less severe moments of stuttering, as well as those using a variety of techniques to mask their stuttering. Using these techniques to such an extent that a person can "pass" as fluent is known as covert or interiorised stuttering. Covert stuttering has been related to increased anxiety and other negative affect, and to self-stigma, ${ }^{61,62}$ but can also be understood as an active form of resistance against stigmatisation by others. ${ }^{63}$ In short, it is not possible to use moments of 
stuttering alone as a metric to quantify the experience of stuttering, and it is clearly not the case that those people who overtly stutter the least are necessarily impacted least by living with a stutter. It is not currently clear what combination of behaviours are captured in imaging studies of the stuttering trait, or state. So, it would be helpful to quantify compensatory strategies and covertness in future studies with people who stutter, to better understand patterns of stuttering and their neural underpinnings.

\section{Summary}

We have previously shown that combining tDCS with a behavioural intervention using metronome and choral speech improved speech fluency in adult men who stutter, measured one and six weeks later. ${ }^{8}$ Here we found that the group who had received tDCS also showed significantly increased brain activity one week after the intervention, relative to the sham group, in the cortical speech network and subcortical regions. Activation increases were found in left inferior frontal cortex, which was located underneath the anode during stimulation, as well as in connected regions of the network supporting fluent speech production. This suggests that tDCS, in combination with temporary fluency-enhancing speaking tasks, promoted a lasting change in the speech network engaged during fluent speech production. In addition, the tDCS intervention supported the activation of the basal ganglia-thalamocortical motor circuit which has been implicated in developmental stuttering.

\section{Acknowledgements}

We would like to thank all the participants who took part in this study. We would also like to thank Lisa Bruckert, Nicola Filipinni, Jane Francis and Steven Knight for their assistance with fMRI data collection, Anthony Chesters and Charlotte Coyte for their assistance with speech data collection and coding, and Faraneh Vargha-Khadem for access to testing facilities for tDCS sessions. 


\section{Funding}

A Medical Research Council UK Clinical Research Training Fellowship, MR/K023772/1, to J.C., and a Medical Research Council UK Career Development Fellowship, G1000566, to R.M., and a British Academy/Leverhulme Trust small project grant SG130103 to KW funded this work.

\section{Competing interests}

The authors report no competing interests. 


\section{References}

1. Iverach L, Menzies RG, O'Brian S, Packman A, Onslow M. Anxiety and stuttering: continuing to explore a complex relationship. Am J Speech Lang Pathol. 2011;20(3):221-232.

2. Iverach $\mathrm{L}, \mathrm{O}$ 'Brian $\mathrm{S}$, Jones $\mathrm{M}$, et al. The five factor model of personality applied to adults who stutter. Journal of communication disorders. 2010;43(2):120-132.

3. Craig A, Blumgart E, Tran $\mathrm{Y}$. The impact of stuttering on the quality of life in adults who stutter. Journal of Fluency Disorders. 2009;34(2):61-71.

4. O'Brian S, Jones M, Packman A, Menzies R, Onslow M. Stuttering severity and educational attainment. J Fluency Disord. 2011;36(2):86-92.

5. Klein JF, Hood SB. The impact of stuttering on employment opportunities and job performance. J Fluency Disord. 2004;29(4):255-273.

6. Johnson $M$, Baxter $S, B$ lank $L$, et al. The state of the art in non-pharmacological interventions for developmental stuttering. Part 2: qualitative evidence synthesis of views and experiences. Int J Lang Commun Disord. 2016;51(1):3-17.

7. Brignell A, Krahe M, Downes M, Kefalianos E, Reilly S, Morgan AT. A systematic review of interventions for adults who stutter. J Fluency Disord. 2020;64:105766.

8. Chesters J, Mottonen R, Watkins KE. Transcranial direct current stimulation over left inferior frontal cortex improves speech fluency in adults who stutter. Brain. 2018;141(4):1161-1171.

9. Watkins KE, Smith SM, Davis S, Howell P. Structural and functional abnormalities of the motor system in developmental stuttering. Brain : a journal of neurology. 2008;131(Pt 1):50-59.

10. Connally EL, Ward D, Pliatsikas C, et al. Separation of trait and state in stuttering. Hum Brain Mapp. 2018;39(8):3109-3126.

11. Xuan $\mathrm{Y}$, Meng $\mathrm{C}$, Yang $\mathrm{Y}$, et al. Resting-state brain activity in adult males who stutter. Plos One. 2012;7(1):e30570.

12. Chang SE, Angstadt M, Chow HM, et al. Anomalous network architecture of the resting brain in children who stutter. J Fluency Disord. 2018;55:46-67.

13. Budde KS, Barron DS, Fox PT. Stuttering, induced fluency, and natural fluency: A hierarchical series of activation likelihood estimation meta-analyses. Brain and Language. 2014;139:99-107.

14. Belyk M, Kraft SJ, Brown S. Stuttering as a trait or state - an ALE meta-analysis of neuroimaging studies. The European journal of neuroscience. 2015;41(2):275-284.

15. Wu JC, Maguire G, Riley G, et al. A positron emission tomography [18F]deoxyglucose study of developmental stuttering. NeuroReport. 1995;6(3):501-505.

16. Fox PT, Ingham RJ, Ingham JC, et al. A PET study of the neural systems of stuttering. Nature. 1996;382(6587):158-161.

17. Neumann K, Preibisch C, Euler HA, et al. Cortical plasticity associated with stuttering therapy. Journal of Fluency Disorders. 2005;30(1):23-39.

18. Toyomura A, Fujii T, Kuriki S. Effect of external auditory pacing on the neural activity of stuttering speakers. Neurolmage. 2011;57(4):1507-1516. 
19. Sommer M, Koch MA, Paulus W, Weiller C, Buchel C. Disconnection of speechrelevant brain areas in persistent developmental stuttering. Lancet. 2002;360(9330):380-383.

20. Chang SE, Erickson KI, Ambrose NG, Hasegawa-Johnson MA, Ludlow CL. Brain anatomy differences in childhood stuttering. Neurolmage. 2008;39(3):1333-1344.

21. Neef NE, Anwander A, Friederici AD. The Neurobiological Grounding of Persistent Stuttering: from Structure to Function. Curr Neurol Neurosci Rep. 2015;15(9):63.

22. Watkins K. Developmental disorders of speech and language: from genes to brain structure and function. Prog Brain Res. 2011;189:225-238.

23. Chang SE, Horwitz B, Ostuni J, Reynolds R, Ludlow CL. Evidence of left inferior frontal-premotor structural and functional connectivity deficits in adults who stutter. Cereb Cortex. 2011;21(11):2507-2518.

24. Neef NE, Hoang TN, Neef A, Paulus W, Sommer M. Speech dynamics are coded in the left motor cortex in fluent speakers but not in adults who stutter. Brain : a journal of neurology. 2015.

25. Bohland JW, Bullock D, Guenther FH. Neural representations and mechanisms for the performance of simple speech sequences. Journal of Cognitive Neuroscience. 2010;22(7):1504-1529.

26. Brown S, Ingham RJ, Ingham JC, Laird AR, Fox PT. Stuttered and fluent speech production: an ALE meta-analysis of functional neuroimaging studies. Human Brain Mapping. 2005;25(1):105-117.

27. Toyomura A, Fujii T, Kuriki S. Effect of an 8-week practice of externally triggered speech on basal ganglia activity of stuttering and fluent speakers. Neurolmage. 2015.

28. Alm PA. Stuttering and the basal ganglia circuits: a critical review of possible relations. Journal of communication disorders. 2004;37(4):325-369.

29. Civier O, Bullock D, Max L, Guenther FH. Computational modeling of stuttering caused by impairments in a basal ganglia thalamo-cortical circuit involved in syllable selection and initiation. Brain Lang. 2013;126(3):263-278.

30. Glickstein M, Stein J. Paradoxical movement in Parkinson's disease. Trends Neurosci. 1991;14(11):480-482.

31. Caligiore D, Mannella F, Arbib MA, Baldassarre G. Dysfunctions of the basal gangliacerebellar-thalamo-cortical system produce motor tics in Tourette syndrome. PLoS Comput Biol. 2017;13(3):e1005395.

32. Smits-Bandstra S, De Nil LF. Sequence skill learning in persons who stutter: implications for cortico-striato-thalamo-cortical dysfunction. J Fluency Disord. 2007;32(4):251-278.

33. Giraud AL, Neumann K, Bachoud-Levi AC, et al. Severity of dysfluency correlates with basal ganglia activity in persistent developmental stuttering. Brain and Language. 2008;104(2):190-199.

34. Davidow JH. Systematic studies of modified vocalization: the effect of speech rate on speech production measures during metronome-paced speech in persons who stutter. Int J Lang Commun Disord. 2014;49(1):100-112.

35. Kalinowski J, Saltuklaroglu T. Speaking with a mirror: engagement of mirror neurons via choral speech and its derivatives induces stuttering inhibition. Medical Hypotheses. 2003;60(4):538-543.

36. Nombela C, Hughes LE, Owen AM, Grahn JA. Into the groove: can rhythm influence Parkinson's disease? Neurosci Biobehav Rev. 2013;37(10 Pt 2):2564-2570. 
37. Riley G. Stuttering Severity Instrument. 4th ed. Austin, TX: Pro-ed; 2009.

38. Measurements ISOS. IEEE Recommended Practice for Speech Quality Measurements. leee T Acoust Speech. 1969;Au17(3):225-246.

39. Jenkinson M, Bannister P, Brady M, Smith S. Improved optimization for the robust and accurate linear registration and motion correction of brain images. Neuroimage. 2002;17(2):825-841.

40. Jenkinson $\mathrm{M}, \mathrm{Smith} \mathrm{S}$. A global optimisation method for robust affine registration of brain images. Med Image Anal. 2001;5(2):143-156.

41. Smith SM. Fast robust automated brain extraction. Hum Brain Mapp. 2002;17(3):143-155.

42. Leech R, Braga R, Sharp DJ. Echoes of the brain within the posterior cingulate cortex. J Neurosci. 2012;32(1):215-222.

43. Winkler AM, Ridgway GR, Webster MA, Smith SM, Nichols TE. Permutation inference for the general linear model. Neuroimage. 2014;92:381-397.

44. Patenaude B, Smith SM, Kennedy DN, Jenkinson M. A Bayesian model of shape and appearance for subcortical brain segmentation. Neuroimage. 2011;56(3):907-922.

45. De Nil LF, Kroll RM, Lafaille SJ, Houle S. A positron emission tomography study of short- and long-term treatment effects on functional brain activation in adults who stutter. Journal of Fluency Disorders. 2003;28(4):357-379; quiz 379-380.

46. Ingham RJ, Grafton ST, Bothe AK, Ingham JC. Brain activity in adults who stutter: similarities across speaking tasks and correlations with stuttering frequency and speaking rate. Brain Lang. 2012;122(1):11-24.

47. Neef NE, Anwander A, Butfering C, et al. Structural connectivity of right frontal hyperactive areas scales with stuttering severity. Brain. 2018;141(1):191-204.

48. Krainik A, Lehericy $\mathrm{S}$, Duffau $\mathrm{H}$, et al. Postoperative speech disorder after medial frontal surgery: role of the supplementary motor area. Neurology. 2003;60(4):587594.

49. Ziegler W, Kilian B, Deger K. The role of the left mesial frontal cortex in fluent speech: evidence from a case of left supplementary motor area hemorrhage. Neuropsychologia. 1997;35(9):1197-1208.

50. Moller J, Jansma BM, Rodriguez-Fornells A, Munte TF. What the brain does before the tongue slips. Cereb Cortex. 2007;17(5):1173-1178.

51. Paus T. Primate anterior cingulate cortex: where motor control, drive and cognition interface. Nat Rev Neurosci. 2001;2(6):417-424.

52. Dick AS, Garic D, Graziano P, Tremblay P. The frontal aslant tract (FAT) and its role in speech, language and executive function. Cortex. 2019;111:148-163.

53. Kronfeld-Duenias V, Amir O, Ezrati-Vinacour R, Civier O, Ben-Shachar M. The frontal aslant tract underlies speech fluency in persistent developmental stuttering. Brain Struct Funct. 2016;221(1):365-381.

54. Cler GJ, Krishnan S, Papp D, Wiltshire CEE, Chesters J, Watkins KE. Elevated iron concentration in putamen and cortical speech motor network in developmental stuttering. Brain. 2021;144(10):2979-2984.

55. Lu C, Chen C, Ning N, et al. The neural substrates for atypical planning and execution of word production in stuttering. Experimental neurology. 2010;221(1):146-156.

56. Zeharia N, Hertz U, Flash T, Amedi A. New whole-body sensory-motor gradients revealed using phase-locked analysis and verified using multivoxel pattern analysis and functional connectivity. J Neurosci. 2015;35(7):2845-2859. 
57. Wu JC, Maguire G, Riley G, et al. Increased dopamine activity associated with stuttering. Neuroreport. 1997;8(3):767-770.

58. Ingham RJ, Wang Y, Ingham JC, Bothe AK, Grafton ST. Regional brain activity change predicts responsiveness to treatment for stuttering in adults. Brain Lang. 2013;127(3):510-519.

59. Neumann K, Euler HA, von Gudenberg AW, et al. The nature and treatment of stuttering as revealed by $\mathrm{fMRI} A$ within- and between-group comparison. Journal of Fluency Disorders. 2003;28(4):381-409; quiz 409-410.

60. Menke $\mathrm{R}$, Meinzer $\mathrm{M}$, Kugel $\mathrm{H}$, et al. Imaging short- and long-term training success in chronic aphasia. BMC Neurosci. 2009;10:118.

61. Douglass JE, Schwab M, Alvarado J. Covert Stuttering: Investigation of the Paradigm Shift From Covertly Stuttering to Overtly Stuttering. Am J Speech Lang Pathol. 2018;27(3S):1235-1243.

62. Boyle MP. Assessment of stigma associated with stuttering: development and evaluation of the self-stigma of stuttering scale (4S). J Speech Lang Hear Res. 2013;56(5):1517-1529.

63. Constantino CD, Manning WH, Nordstrom SN. Rethinking covert stuttering. J Fluency Disord. 2017;53:26-40. 